\title{
WNN Tolerance Fault Diagnosis for Analog Circuits Based on Wavelet Packet Transform Features
}

\author{
Haiqing Huang ${ }^{1, a}$, Shiyao Ren $^{2, b}$, Nan Yang ${ }^{2, c}$ \\ ${ }^{1}$ Xijing College, Xi’an, Shaanxi, 710123, China \\ ${ }^{2}$ Air Traffic Control and Navigation College, Air Force Engineering, University, Xi’an, Shaanxi, 710051, \\ China \\ à huang122@163.com, brensy888@sina.com, c yangn001@126.com
}

Keywords: WNN; Wavelet packet transform; Analog circuit; Fault diagnosis; Feature extraction

\begin{abstract}
For the sample construction of Wavelet Neural Network (WNN) fault diagnosis modeling for analog circuits, a feature extraction method based on Wavelet Packet Transform (WPT) is proposed. The simulation results on Sallen-Key band-pass filter show that, compared with Multi-Resolution Transform, WPT has better feature extraction ability for the tolerance faults of analog circuits, and can improve the convergence rate and diagnosis correct rate of WNN.
\end{abstract}

\section{Introduction}

The paper focuses on feature extraction for WNN [1][2] application of tolerance fault diagnosis for analog circuits. Based on this, Wavelet Packet Transform (WPT) is introduced in building Wavelet Neural Network (WNN) model to extract the feature of tolerance faults for analog circuits.

\section{Wavelet Packet Transform (WPT)}

In fact, for many problems, it is only necessary to extract the information of points at specific time and frequency. At the point in specific frequency domain, the resolution of frequency domain needs to be improved as much as possible; at the point in specific time domain, the resolution of time domain needs to be improve as much as possible. But MRT cannot satisfy this requirement. This is because MRT only decomposes $V$ (scale) space, i.e. $V_{0}=V_{1} \oplus W_{1}=V_{2} \oplus W_{2} \oplus W_{1}=\cdots$, and doesn't further decompose $W$ (space). WPT can further decompose $W_{j}$, which can satisfy above requirements with more extensive application.

WPT, derived on the basis of wavelet transform, is a generalization of the wavelet concept [3][4]. The definition of wavelet packet is given as follows:

For given orthogonal scaling function $\phi(t)$ and wavelet function $\psi(t)$, its two-scale relationship

$$
\begin{aligned}
& \phi(t)=\sqrt{2} \sum_{k} h_{0 k} \phi(2 t-k) \\
& \psi(t)=\sqrt{2} \sum_{k} h_{1 k} \psi(2 t-k)
\end{aligned}
$$

where $h_{0 k}, h_{1 k}$ are the filter coefficients in MRT.

Then define the following recursive relationship

$$
\begin{aligned}
& w_{2 n}(t)=\sqrt{2} \sum_{k \in Z} h_{0 k} w_{n}(2 t-k) \\
& w_{2 n+1}(t)=\sqrt{2} \sum_{k \in Z} h_{1 k} w_{n}(2 t-k)
\end{aligned}
$$


where $h_{0 k}, h_{1 k}$ are still the filter coefficient in Eq. (1) and Eq. (2). When $n=0, w_{0}(t)=\phi(t)$ and $w_{1}(t)=\phi(t)$. The function set $\left\{w_{n}(t)\right\}_{k \in Z}$ defined above is the wavelet packet determined by $w_{0}(t)=\phi(t)$.

For the signal $f(t)$, the recursive algorithm of its WPT coefficient is

$$
\left\{\begin{array}{l}
d_{k}^{j+1,2 n}=\sum_{l} h_{0(2 l-k)} d_{k}^{j, n} \\
d_{k}^{j+1,2 n+1}=\sum_{l} h_{1(2 l-k)} d_{k}^{j, n}
\end{array}\right.
$$

The reconstruction algorithm of its wavelet packet is

$$
d_{k}^{j, n}=\sum_{k}\left[h_{0(l-2 k)} d_{k}^{j+1,2 n}+h_{1(l-2 k)} d_{k}^{j+1,2 n+1}\right]=\sum_{k} g_{0}(l-2 k) d_{k}^{j+1,2 n}+\sum_{k} g_{1}(l-2 k) d_{k}^{j+1,2 n+1}
$$

A three-layer wavelet packet decomposition tree can be used to help the intuitive understanding of wavelet packet analysis, as shown in Fig. 1, where A denotes the low frequency part, D denotes the high frequency part, $k$ denotes the level number of wavelet packet decomposition. Then the decomposition relation is:

$$
S=A A A_{3}+D A A_{3}+A D A_{3}+D D A_{3}+A A D_{3}+D A D_{3}+A D D_{3}+D D D_{3}
$$

As far as WPT is concerned, it can be understood that, at each operation, two decimation one operation is simultaneously performed for the high-frequency part and low-frequency part of previous decomposition result (that is, the even or odd part is retained), so that the low frequency band and the high frequency band can have consistent time-frequency resolution. Before the maximum number of decomposition layers is reached, the spatial decomposition mode is conducted all the way to get higher resolution in frequency and time domain space. All frequency bands of last decomposition result are processed according to certain criteria, and then the reconstruction is conducted along reverse way.

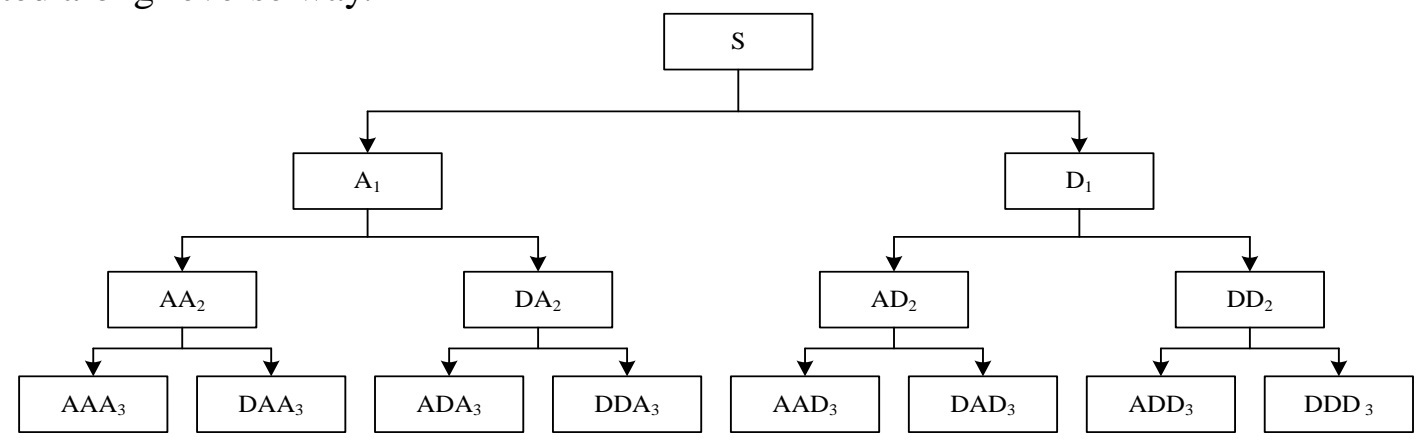

Fig. 1 Structure of three-layer wavelet packet decomposition tree

\section{WPT realization process}

The output signal waveform of analog circuit contains rich frequency components. When the circuit fault occurs, these frequency components are affected by the fault to varying degrees. Some are weakened, others are enhanced. Therefore, the frequency components under fault state are different from that under normal state, that is, its characteristics in amplitude-frequency and phasefrequency have changed, resulting in the change of wavelet coefficient with same frequency components. Usually, the energy of the enhanced frequency component increase, and the energy of the weakened frequency component decrease, and the energy change of different frequency components is significantly different. It can be seen that, the output signal waveform of analog circuit contains a wealth of fault information, and the energy change of some frequency components can make the fault feature of insignificant signals appear with significant change form in wavelet packet 
subspace under different resolutions. These features extracted can characterize the fault of a component in the circuit. Thus the wavelet energy of output signal can be used as the fault feature vector to construct the sample set. The method only needs to carry out fault diagnosis according to the change law of wavelet energy of output signal without solving differential equations [4]. The steps of sample construction based on WPT are as follows:

1) Carry out $j$-th layer wavelet packet decomposition for the fault output signal $f(t)$, and take out coefficient sequences of each frequency component in $j$-th layer respectively, obtaining $2^{j}$, namely $\left\{W_{j}^{0}, W_{j}^{1}, \cdots, W_{j}^{2^{j}-1}\right\}$.

2) Suppose that $S_{j 0}$ denotes the reconstructed signal of $W_{j 0}, S_{j 1}$ denotes the reconstructed signal of $W_{j 1}$, and the like, then

$$
S=S_{j 0}+S_{j 1}+S_{j 2}+\cdots+S_{j\left(2^{j}-1\right)}
$$

3) Suppose that the energy of $S_{j i}$ is $E_{j i}\left(i=0,1,2, \cdots, 2^{j}-1\right)$, then

$$
E_{j i}=\int\left|S_{j i}\right|^{2} d t=\sum_{k=1}^{n}\left|X_{i k}\right|^{2}
$$

where $X_{i k}\left(k=0,1,2, \cdots, 2^{j}-1\right)$ denotes the magnitude of discrete points of reconstructed signal $S_{j i}$.

4) The energy is taken as $2^{j}$-dimension vector of the element

$$
P=\left[E_{j}^{0}, E_{j}^{1}, E_{j}^{2}, \cdots, E_{j}^{2^{j}-1}\right]
$$

Generally, the energy of each component of $P$ is not in the same dimension. Therefore, $P$ needs to be normalized before it can be used as the feature vector of the fault, and is combined with the expected output vector to obtain the sample set of WNN. It should be noted that, the wavelet basis function and the number of layers of wavelet packet decomposition should be appropriately selected according to the characteristics of fault signals.

\section{Experiment example}

Sallen-Key bandpass filter is selected as the research subject, as shown in Fig. 2. The output "Out” is only test node. Through AC small-signal sensitivity analysis, it can determine that the fault set contains 8 fault modes, namely $\mathrm{R}_{2^{-}}, \mathrm{R}_{2}+, \mathrm{R}_{3^{-}}, \mathrm{R}_{3}+, \mathrm{C}_{1^{-}}, \mathrm{C}_{1}+, \mathrm{C}_{2^{-}}, \mathrm{C}_{2}+$, where the symbol ' $+^{\text {' }}$ and '-' represent too large tolerance fault and too small tolerance fault respectively. In this way, 8 fault modes together with normal mode add up to 9 modes. In addition, "0-l” method can be used to obtain the expected output vector of 8 output nodes in WNN.

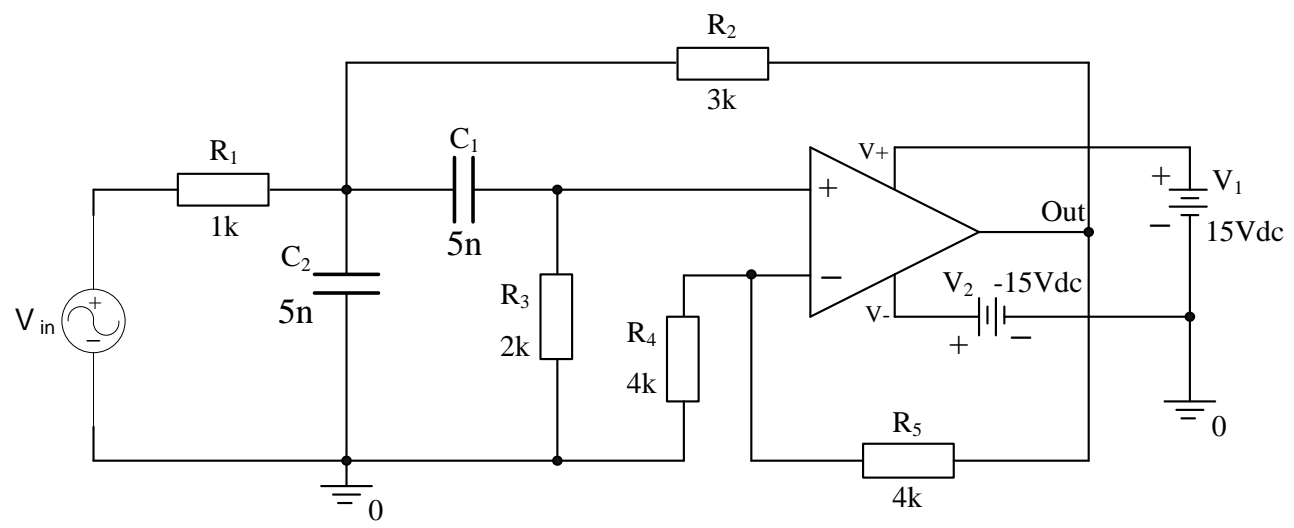

Fig. 2 Sallen-Key bandpass filter

In Fig. 1, the input power source $V_{\text {in }}$ is set as sine wave input excitation in the circuit, the voltage 
amplitude is $4 \mathrm{~V}$, the voltage frequency is $1 \mathrm{KHz}$, the start time of scan is $0.25 \mathrm{~ms}$, the end time of scan is $1.5 \mathrm{~ms}$, the step size of scan is $0.5 \mu \mathrm{s}$. According to the feature of each mode, the fault model is set for the fault component. Each component without the fault is in the range of normal tolerance respectively. The characteristic analysis of the circuit is set as the transient analysis. Monte Carlo analysis is conducted through software ORCAD. In this way, the output waveform under $\mathrm{R}_{2}$ - fault mode can be obtained as shown in Fig. 3, where the dotted line denotes the voltage frequency response waveform of the output of normal signal.

It can be seen from the literature [4] that, $\mathrm{dbN}$ wavelet satisfies the conditions such as orthogonality, tight support and regularity with good time-frequency localization, which can describe the essential characteristics of the signal more finely. In $\mathrm{dbN}$ wavelets, $\mathrm{db} 2, \mathrm{db} 3$ and $\mathrm{db} 5$ wavelets are commonly used. For the feature extraction of tolerance fault for analog circuit, compared with db3 and db5 wavelets, db2 wavelet can better reflect the characteristics of the signal and extract more mutation features. Therefore, in the paper, $\mathrm{db} 2$ is selected as the wavelet basis function of WPT, and can theoretically get better feature extraction effect. For 450 fault mode signals obtained, 3-layer wavelet packet decomposition is first carried out, and then the energy of the 8 bands in the 3rd layer is calculated according to the steps of WPT given in Section 4, and normalized to form an 8-dimensional input vector, finally the obtained input vector can be combined with the corresponding expected output vector to get 450 samples where 180 samples are randomly selected for WNN training and 270 samples remaining for testing WNN model. The modeling capabilities of WNN under feature extraction using MRT (Multi-Resolution Transform) and WPT are compared in Table 1, where IDN is incorrect diagnosis number, CIN is convergence iteration number.

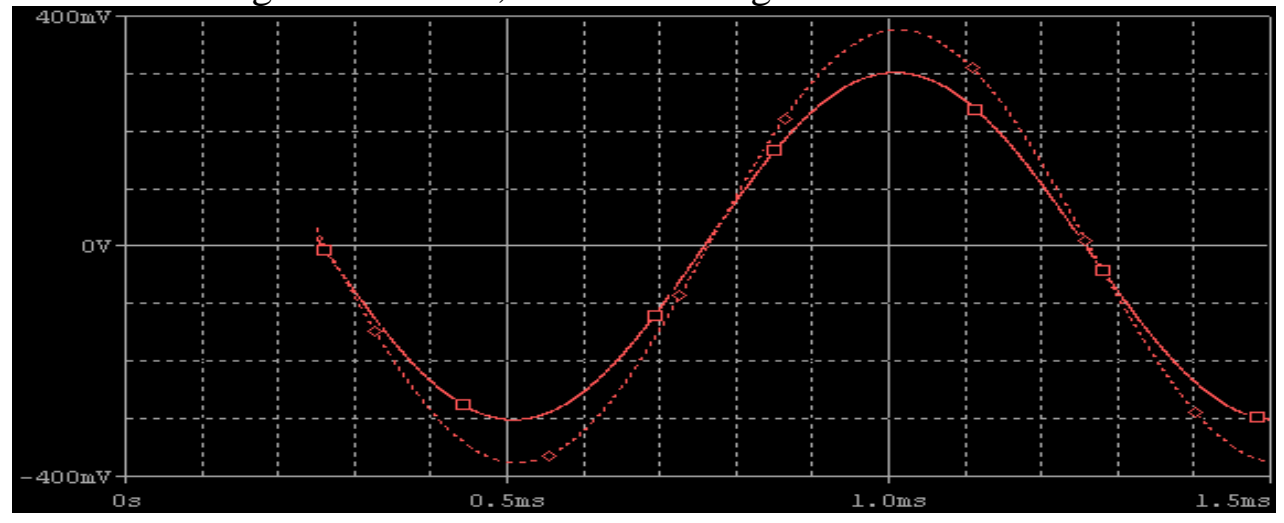

Fig. 3 Output waveform under $\mathrm{R}_{2}$ - mode

Table 1 Comparison of WNN modeling under MRT and WPT feature extraction

\begin{tabular}{ccccccccc}
\hline \multirow{2}{*}{$\begin{array}{c}\text { Hidden } \\
\text { nodes } \\
\text { number }\end{array}$} & $\begin{array}{c}\text { Training } \\
\text { IDN }\end{array}$ & $\begin{array}{c}\text { Test } \\
\text { IDN }\end{array}$ & CIN & & $\begin{array}{c}\text { Training } \\
\text { IDN }\end{array}$ & $\begin{array}{c}\text { Test } \\
\text { IDN }\end{array}$ & CIN \\
\hline 10 & 21 & 28 & 478 & & 23 & 24 & 325 \\
15 & 22 & 25 & 334 & & 21 & 23 & 275 \\
20 & 25 & 31 & 428 & & 25 & 28 & 359 \\
\hline
\end{tabular}

\section{Conclusions}

In contrast with the use of the fault features extracted by MRT, the use of the fault features extracted by WPT can achieve the better effect of tolerance fault diagnosis for analog circuits, which is very important for the wider application of WNN. In summary, on the basis of the features of tolerance fault extracted by WPT, the tolerance fault diagnosis based on WNN is feasible and effective for analog circuit. 


\section{References}

[1] Q. H. Zhang, A. Benveniste. Wavelet network. IEEE Transactions on Neural Networks, 3(6), 1992, 889-898

[2] Q. H. Zhang. Using wavelet network in nonparametric estimation. IEEE Transactions on Neural Networks, 8(2),1997, 227-236

[3] F. Argüello, J. López, M. A. Trenas, E. L. Zapata. Architecture for wavelet packet transform based on lifting steps. Parallel Computing, 28(7-8), 2002, 1023-1037

[4] M. H. Gao. The research of analog circuit soft fault diagnosis based on wavelet analysis and neural network. Master Degree Dissertation, 2009 